\title{
Reactive Thrombocytosis
}

\author{
Poul Guldfeldt ${ }^{\mathrm{a}, \mathrm{b}}$
}

\begin{abstract}
This case report is about a patient who went through a small abdominal operation and afterwards was diagnosed with thrombocytosis. It was found retrospectively that the patient for some time had had thrombocytosis. The patient was referred to the haematological department for further testing. There was no evidence of myeloproliferative disease, instead there was found a $13 \mathrm{~mm}$. malignancy suspect infiltrate located to the base of the right lung. The patient went through curative surgery. However there was found a small metastasis in a lymph node and the patient was given adjuvant chemotherapy, 3 months postoperatively, there is still decreasing platelets of $535 \times 10^{9} / \mathrm{L}$ and the patient is considered cured.
\end{abstract}

Keywords: Thrombocytosis; Malignancy; Metastasis

\section{Introduction}

The clinically important message of this case report is that a relatively common cancer disease such as non small cell lung cancer can have its first manifestations in a high platelet count. The pathogenesis of this is described in the discussion part. It is a relatively rare first manifestation of non small cell lung cancer.

When making rounds on a surgical ward it is often seen that the platelet count can be high after major surgery. This was not the case here. This patient had only been through a small abdominal procedure to cure a hernia. The high platelet count seemed odd considering the small abdominal pro-

Manuscript accepted for publication February 21, 2013

${ }^{a}$ Department of surgery, Hospital of Southern Jutland, Denmark

${ }^{\mathrm{b}}$ Corresponding address: Kirurgisk afdeling, Sygehus Sonderjylland, Kresten Philipsens Vej 156200 Aabenraa, Denmark.

Email: poulguldfeldt@hotmail.com

doi: http://dx.doi.org/10.4021/jmc1158e cedure and it was interpreted as an indicator for underlying disease. The patient had no symptoms from any of her organs and the remaining blood work was normal.

After thorough testing, it turned out to be cancer of the right lung causing the high platelet count. Three months after the high platelet count was discovered the patient was cured for the life threatening disease she did not have any symptoms of and which was diagnosed by a coincidence. The platelet count was at this time decreasing and almost normalized.

\section{Case Report}

A 62-year-old woman with an incisional hernia was admitted to the General Hospital for planned hernia surgery. The operation was conducted laparoscopic and without complications. The patient was discharged well-being the next day.

On discharge there was found thrombocytosis at $819 \times$ $10^{9} / \mathrm{L}$ and it was retrospectively realized that the patient already at a preoperative consultation 2 months before had had thrombocytosis of $833 \times 10^{9} / \mathrm{L}$.

Contacts were made to the haematological department for further testing on suspicion of essential thrombocytosis. The patient was also started in the low-dose aspirin.

In the haematological department there were performed bone marrow examination and JAK 2 analysis. These studies did not prove to support the suspicion of myeloproliferative disease.

As part of the standard examination program there was performed an abdominal ultrasound and a chest X-ray was produced.

The chest $\mathrm{x}$-ray reviled a malignant suspicious infiltrate of the lower part of the right lung. The patient subsequently entered into the investigation protocol for primary lung cancer and a computed tomography of the chest was performed which increased the suspicion of cancer (Fig. 1). The infiltrate had a size of $13 \mathrm{~mm}$ in diameter. In addition a computed axial tomography - guided biopsy was performed. However this procedure was complicated by a small pneumothorax which was conservatively treated.

The biopsy revealed adenocarcinoma CK 7 and TTF 1 


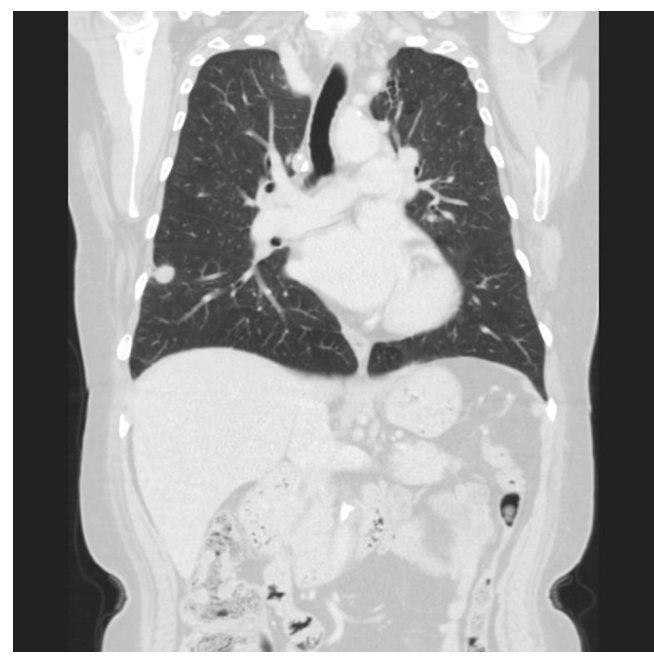

Figure 1. The chest $x$-ray revealed a malignant suspicious infiltrate of the lower part of the right lung.

positive, thus compatible with primary lung cancer.

A positron emission tomography - computed tomography did not raise suspicion of disseminated disease and as a lung function examination revealed an FEV1 of $99 \%$ of predicted and an FVC of $96 \%$ of predicted, the patient was referred to the university hospital for radical surgery.

The tumor was removed in toto and the histology report showed adenocarcinoma. There was found one metastases in a lymph node of the medial bronchus and it was therefore necessary with adjuvant chemotherapy.

\section{Discussion}

Reactive thrombocytosis can be seen as part of an acute inflammatory condition, after major surgery and in cancer diseases. Elevated values are also seen among patients suffering from polycythemia, chronic myeloid leukemia, acute infections and chronic inflammatory conditions [1].

The reason for thrombocytosis in malignancies is not yet fully understood. One possible explanation may be associated with the formation of megakaryocytes which can be stimulated by interleukin-6. It is known that interleukin- 6 can be released from the tumor cells both in vitro and in vivo, and thus stimulating the formation of megakaryocytes resulting in thrombocytosis $[2,3]$.

There is usually no indication for measuring platelet numbers postoperatively, unless there has been a greater blood loss or if the patient has an increased risk of bleeding. None of these causes was present here. By mistake the platelet numbers was measured postoperatively and thus, it was quite by accident that it was recognized that the patient had thrombocytosis at discharge.

When a doctor discovers that a patient has elevated platelet counts some of the first questions are why they are high at what should I do.

Reactive thrombocytosis is usually no indication for initiation of aspirin. In reactive thrombocytosis with values up to $1,000 \times 10^{9} / \mathrm{L}$ there is no evidence of any benefit from treatment with aspirin because the risk of a thromboembolic complication is very low. Some studies have shown a risk as low as $1.6 \%$ for a thromboembolic complication of reactive thrombocytosis. This is why it is generally not recommended to treat with aspirin for prophylactic purposes in patients with reactive thrombocytosis [1].

If there is pronounced thrombocytosis with platelet counts of $1,000 \times 10^{9} / \mathrm{L}$ or higher the patient will suffer from an acquired von Willebrand factor deficiency. In cases with extreme high platelet counts there will be too many platelets in relation to von Willebrand factor. This will result in an increased bleeding tendency and starting aspirin will aggravate this condition [1].

There are various data on the frequency of thrombocytosis in patients with non small cell lung cancer and it is estimated that between $4.15 \%$ and $32.1 \%$ have thrombocytosis at the time of diagnosis. These different observations may be because the patients were at different stages of their disease [3-5].

However, there is general agreement that reactive thrombocytosis as mono symptom and first symptom is rare and reactive thrombocytosis at baseline indicates a poorer prognosis [3-5].

When it comes to the doctors obligations it is recommended that a thorough investigation like the one this patient had is performed.

Many conditions can lead to elevated platelet counts but cancer is obvious the most feared. If the doctor makes a routine screening program for the most ordinary cancer conditions adapted to the hospital standards he is working under a great deal has been done for the patient.

\section{Conflict of Interest}

There is no conflict of interest.

\section{References}

1. Bleeker JS, Hogan WJ. Thrombocytosis: diagnostic evaluation, thrombotic risk stratification, and risk-based management strategies. Thrombosis. 2011;2011:536062.

2. Alexandrakis MG, Passam FH, Perisinakis K, Ganotakis E, Margantinis G, Kyriakou DS, Bouros D. Serum proinflammatory cytokines and its relationship to clinical parameters in lung cancer patients with reactive thrombocytosis. Respir Med. 2002;96(8):553-558.

3. Pedersen LM, Milman N. [The prognostic value of 
thrombocytosis in patients with primary lung cancer]. Ugeskr Laeger. 1998;160(26):3917-3920.

4. Ferrigno D, Buccheri G. Hematologic counts and clinical correlates in 1201 newly diagnosed lung cancer patients. Monaldi Arch Chest Dis. 2003;59(3):193-198.
5. Tomita M, Shimizu T, Ayabe T, Onitsuka T. Prognostic significance of the combined use of preoperative platelet count and serum carcinoembryonic antigen level in nonsmall-cell lung cancer. Gen Thorac Cardiovasc Surg. 2010;58(11):573-576. 